\title{
Research on Key Welding Technologies of Aluminum Matrix Composites
}

\author{
Qiaofang Fan, Yanchuan Yang, Ming Wang \\ Jiangsu College of Safety Technology, Xuzhou, 221011, China
}

Keywords: Welding Technologies, Aluminum Matrix Composites, SiC

\begin{abstract}
Aluminum matrix composite is one of the most commonly used compounds in chemical industry. In this paper, the main welding technologies of aluminum matrix composites are introduced, including the laser welding technology, the friction welding technology and the diffusion welding technology. Aluminum matrix composites reinforced by particle have many advantages, such as high specific strength and modulus, corrosion resistance, radiation resistance and dimensional stability. In this paper, the technology of $\mathrm{SiC}$ particle reinforced aluminum matrix composites is also discussed to provide some references for the relative researchers.
\end{abstract}

\section{Introduction}

Aluminum matrix composite is the commonly used industrial pure aluminum, aluminum, aluminum, aluminum and other aluminum matrix composite material, usually in some with high strength, high modulus, wear resistance and high temperature properties of particles, whiskers or fibers. By enhancing the different aluminum based composite materials can be divided into fiber reinforced and non-continuous enhancement type compared to two categories. However, production of fiber reinforced aluminum matrix composites technology is complex, the cost is relatively high the application is only limited to aviation, aerospace, military and other minority areas. Instead of continuously reinforced aluminum matrix composites due to solve the problem of high cost of fiber reinforced by fiber reinforced aluminum matrix composites, the manufacturing process is simple, mainly used in powder metallurgy and casting two manufacturing methods; low cost of raw materials, through drawing, rolling and forging process and other conventional methods for further processing; due to the enhanced phase in the matrix diffusion is isotropic, it is suitable for under complex stress state; in addition, it also maintains most of the excellent performance, overcome the preparation process such as fiber damage, microstructure inhomogeneity, fiber and fiber in contact with each other, with a big reaction effect of material properties due to many disadvantages. The two processing performances between particles of metal matrix composite short fiber and whisker reinforced and reinforced between continuous fiber reinforced metal matrix composites. In addition, whiskers are potentially harmful to health during operation. So in the first study, discontinuous reinforced the largest and most widely used is $\mathrm{Al}$ based composite materials development in metal matrix composites, and welding composite materials. Aluminum matrix composite particles as the focus of development enhancement is more difficult, which is decided by the special aluminum base composite material of its own.

\section{Primary Methods of Welding Technologies of Aluminum Matrix Composites}

Laser Welding. The welding process of heat conduction, laser radiation heating surface, surface heat through the heat transfer oriented internal diffusion, by controlling the pulse width and energy, peak power and repetition frequency parameters of the melting, forming a melt pool. Because of its unique advantages, it has been successfully used in the precision welding of micro and small parts. As the laser welding with high energy density, high welding speed, weld fine, small heat affected zone 
and easy to realize process automation and other advantages, therefore, from the point of reducing melting zone and heat affected zone, high power laser welding on the connection seems to be very advantageous in metal matrix composites. As the laser of high energy density, caused by welding the local temperature of molten pool is very high, but due to the enhanced phase larger resistivity, the laser absorption rate is high, easily lead to enhanced phase serious overheating, even melting. Effect of composite welding of weld metal in the crystallization process with liquid solid interface by weld metal particle breakage, pulse frequency directly affects the particle breakage and its distribution in the weld metal. The composites are improved with increasing pulse frequency. It is pointed out that the laser pulse frequency is the main factor affecting the particle distribution. Due to the high energy density of laser beam, the heating and cooling speed is very fast, the pool is small, the time is short and the heat input is low. In addition, the characteristics of laser ablation and laser ablation are the essential factors to avoid the excessive damage of the microstructure. Compared with the traditional welding method, laser welding filler is the unique advantages in general in the welding process, so the connection of metal matrix composites is very attractive, has become the hot spot welding of metal matrix composites. Some progress has been made in laser welding of aluminum matrix composites.

Friction Welding. Friction welding is a solid state welding method for welding. Under pressure, pressure is increasing and the effect of torque at constant or under welding using the relative motion between the contacting surface of friction heat and plastic deformation heat in the friction surface and the surrounding region and region near the temperature rises to close to the temperature range but below the melting point, the decrease of deformation resistance and ductility is improved interface, the oxide film is broken, in forging pressure, with the materials to produce plastic deformation and flow through the molecular diffusion and recrystallization and solid welding method welding. Friction welding is suitable for the welding of aluminum matrix composites. Because of the special requirements of the shape. The friction welding is usually suitable for the connection between the simple shape elements such as the tube and bar, and it is difficult to realize the connection between the complex structure and the engineering part. With the increase of friction time, the friction interface temperature and friction torque are almost constant, and the temperature distribution area becomes wider and the flying edge increases gradually. At this stage, the friction pressure and speed are kept constant. When the friction welding area of temperature distribution and deformation to a certain extent, to the brake and make the axial force increases rapidly to the set pressure at the axial shortening rapidly increases, and the interface with the decrease of temperature, friction pressure and friction torque of second peak values, namely after the peak torque. In the process of maintaining pressure and forging after forging process, the weld metal by diffusion and recrystallization, the two sides of the metal is firmly welded together, thus completing the whole welding process. In the whole welding process, the friction interface temperature generally does not exceed the melting point, so the friction welding is a solid state welding.

Diffusion Welding. Diffusion welding is a welding method which makes the welding parts close to each other for a period of time under certain temperature and pressure. Because the welding is usually completed in the high temperature environment, in order to avoid the high temperature oxidation of the weldment. The diffusion welding pressure is small, the workpiece does not produce the macroscopic plastic deformation, and is suitable for the precision parts after welding. Diffusion bonding can be combined with other hot working processes, such as heat dissipation diffusion bonding, powder sintering diffusion bonding and superplastic forming diffusion welding. These combined processes can not only greatly improve productivity, but also solve the problem that cannot be solved by a single process. If the joint performance of various titanium alloys on the supersonic aircraft is the application of superplastic forming - diffusion welding can be made with the same parent material, especially suitable for welding of dissimilar metal materials, graphite and ceramic non-metallic materials, reinforced by high temperature alloys, metal matrix composites and porous sintered materials. Diffusion welding has been widely used in the manufacture of reactor fuel element, honeycomb structure board, electrostatic accelerating tube, all kinds of blades, impeller, die, filter pipe and electronic components. Fiber reinforced metal matrix composite diffusion welding strength mainly depends on the bonding strength between the surface of composite matrix, the matrix metal in 
the whole welding joint percentage of the interface is bigger, the joint strength is higher; and the greater the percentage of fibers, the joint strength will lower. Aluminum matrix composite is mainly diffusion welding with a layer of dense oxide film on the surface of the composite materials, by mechanical or chemical cleaning, it immediately generated, even under high vacuum conditions, this film is difficult to decompose, affect the diffusion between atoms.

\section{Welding Technology of SiC Reinforced Aluminum Matrix Composites}

Preparation Technology of Aluminum Matrix Composites Reinforced by SiC. The pretreatment, cleaning, heating and drying of SiC particles in the resistance furnace heating and melting of substrate material are done. The refining slag, SiC particles reach set temperature behind the stirring while adding treated by metal mound casting, and finally to Aluminum Alloy slab hot extrusion and rolling processing. The results show that the composite billet after hot extrusion, the uniformity of $\mathrm{SiC}$ particles in the matrix increased, and along the extrusion direction was banded obviously. SiC particles are broken and tendency; after rolling, the organization to further refinement, $\mathrm{SiC}$ particle breakage obviously, particle distribution tends to uniform. The tensile strength, yield strength and elongation of the composites increased with the subsequent plastic deformation. The tensile strength, yield strength and elongation of the aluminum matrix composites prepared by stirring casting were improved after hot extrusion and rolling. The subsequent plastic deformation treatment of aluminum matrix composites prepared by stirring casting plays an important role in improving the properties of aluminum matrix composites. The composite material stirring method simple process, low equipment cost, is a method for production of composite material particles mass enhancement; but the mixing composite castings prepared by thick, severe segregation, and because of the mixing process with easy gas, caused by the poor quality of casting. The subsequent plastic deformation of the composites prepared by stirring method plays an important role in improving the microstructure and properties. When the infiltration temperature is between 900 and $1000 \mathrm{DEG} C$, the infiltration speed is fast; when the temperature is between 1000 and 1100 DEG C, the penetration depth increases. With the increase of temperature, the wetting angle between aluminum and $\mathrm{SiC}$ decreases, and the infiltration is easier. Infiltration temperature is too low, the melt viscosity, is not conducive to the infiltration of the preform pore, and is not conducive to play the role of auxiliary infiltration agent; infiltration temperature, infiltration aids can also cause serious loss, the matrix particles thick, infiltration temperature according to different choice of matrix alloy suitable.

Isothermal Deformation Technology. At low strain rate, deformation due to long time, sufficient time dynamic recrystallization and development, while at the high temperature, the recrystallization process in the energy higher, so the nucleation is more significant; while at high strain rates, the compressive dynamic recrystallization time shortened obviously. Very fast, too late to grow grain, therefore the recrystallization grain size is smaller. It is not clear that the recrystallization grains can be seen from the metallographic photograph, but the dynamic recrystallization cannot be ruled out in this case. The temperature, velocity and deformation times of isothermal deformation have an effect on the microstructure of the composites, the distribution of $\mathrm{SiC}$ particles and the room temperature. The change of microstructure and tensile properties of isothermal $\mathrm{SiC}$ aluminum matrix composites after deformation analysis of deformation temperature, deformation and heat will increase the grain growth; accelerate the deformation rate the grain became smaller, and the size distribution of $\mathrm{SiC}$ local aggregation phenomenon. This is because the SiC particles are more easily deformed with the matrix when the deformation is slow, and the SiC particles, which are both hard and brittle particles, are difficult to coordinate with the matrix when the flow velocity is fast. The tensile properties at room temperature changed after isothermal deformation. The deformation of the composite material with large deformation is higher than that of the deformation time, and the effect of the deformation time on the tensile properties is not significant. The results show that the high deformation temperature causes the matrix to undergo recrystallization in the process of plastic deformation and the grain becomes finer, which improves the plasticity of the material. The increase of temperature makes the bonding ability between SiC particles and matrix decrease. 
Heat Treatment Technology. Heat treatment is a kind of metal hot working process in which the material is obtained by means of heating, heat preservation and cooling under the condition of solid state. The metal heat treatment is one of the most important technology in mechanical manufacturing, compared with other processing technology, heat treatment generally does not change the shape of the workpiece and the overall chemical composition, but by changing the internal microstructure, or change the surface chemical composition, gift or improve the performance. Its characteristic is to improve the intrinsic quality of the work piece, which is generally not visible to the naked eye. For $\mathrm{SiCp} / \mathrm{Al}$ composites, due to the addition of ceramic particles, the toughness of the composites is poor, which limits the application of aluminum matrix composites to some extent. The composites were prepared by powder hot extrusion, and the mechanical properties of the extruded and T4 states were compared. Compared with the extruded composites, by T4 state after the treatment rate remained at $7 \%$ level in the elongation and contraction, strength has been greatly improved, the tensile strength and yield strength values were increased by $104 \mathrm{MPa}$ and $114 \mathrm{MPa}$, increase. After the T4 treatment, the strength of the composites increased due to the increase of the strength of the aluminum alloy matrix, and the strengthening of the load transfer strengthening mechanism of the composite material could enhance the stress of the particle. After T4 treatment, the strength of the composites increases greatly, plastic still remain at the same level, and this does not add enhanced Aluminum Alloy matrix body strength increase by the same heat treatment, the ductility decreases contrary to the law. The results show that the fracture of the composites is mainly matrix fracture, and the fracture of the T4 composites is not only the matrix but also the particle breakage. The additional particle fracture under the matrix region near the particle stress weaken or even disappear, reducing the probability of aluminum matrix fracture; at the same time, the plastic deformation limit of particles weakened the aluminum matrix, the matrix is conducive to free plastic deformation.

\section{Conclusion}

SiC particle, which has excellent properties and low cost, reinforces aluminum matrix composites. It is an ideal particle of metal matrix composite. At present, the joining technology of SiC particle reinforced aluminum matrix composites is still immature, which seriously restricts its popularization and application. It is the research focus of the scientific and technological researchers to strengthen the study of various welding processes, mechanisms and so on.

\section{References}

[1] Sun Ruji, Research on friction stir welding of particulate reinforced aluminum matrix composites for automobile [J]. Electric Welding Machine, 2015, 45(3): 81-83+102.

[2] Dai Jun, Liu Zheng, Wang Yang, Zhang Jie, Zhang Yaocheng, Yang Li, Effect of Laser Power on Weld Formation and Microstructure of TiB2 Enhanced Aluminum Matrix Composite [J]. Hot Working Technology, 2016, 45(5): 37-39+43.

[3] $\mathrm{Xu}$ Feng, Weldablity and capacitor discharge welding of SiC reinforced aluminum matrix composites [J]. Light Alloy Fabrication Technology, 2010, 38(4): 48-51+57.

[4] Li Shuzhi, Wang Tiejun, Wang Ling, Research progress of SiC particle reinforced aluminum matrix composites [J]. Powder Metallurgy Industry, 2017, 27(1):64-70. 